\title{
Modeling of the Thermal Conductivity and Thermomechanical Behavior of Diamond Reinforced Composites
}

\author{
Sergio Nogales ${ }^{\mathrm{a}, \mathrm{b}}$, Helmut J. Böhm ${ }^{\mathrm{a}, *}$ \\ ${ }^{a}$ Institute of Lightweight Design and Structural Biomechanics, \\ Vienna University of Technology, Gußhausstr. 27-29, A-1040 Vienna, Austria \\ ${ }^{\mathrm{b}}$ Max Planck Institute for Plasma Physics, \\ Boltzmannstr. 2, D-85748 Garching, Germany
}

\begin{abstract}
Semi-analytical Mori-Tanaka methods and numerical models for studying the overall thermal conduction behavior of metal matrix composites reinforced by diamond particles are presented, special emphasis being put on the effects of finite interfacial conductances. Good agreement between the simulation approaches is obtained and the influence of particle shapes and homogeneous vs. inhomogeneous interfacial conductances on local and global responses is studied. Analogous methods are applied to modeling the elastic and thermoelastoplastic behavior of diamond reinforced metals.
\end{abstract}

Key words: A. Metal-matrix composites, B. Modeling, B. Thermal properties, B. Mechanical properties

PACS: $16.60-\mathrm{Rc}, 81.05-\mathrm{Ni}$ 


\section{Introduction}

Diamond reinforced metal matrix composites (DRMMCs) are promising candidate materials for attaining elevated thermal conductivities [1,2], and they have been identified as holding the potential for breakthrough applications as heat sinks, e.g., in microelectronics. Accordingly, simulation studies of such materials, the overall thermal conduction behavior of which is not only governed by the material properties and the geometrical arrangement of the constituents, but also by the behavior of the interfaces between them [3], are of considerable practical interest.

To obtain a high effective thermal conductivity, pure or low-alloyed aluminum of copper are typically used as the matrices of DRMMCs. As the coefficients of thermal expansion (CTE) of these metals are an order of magnitude higher than the CTE of the diamonds, substantial thermal stresses must be expected as consequences of production-related cooling-down processes and of temperature excursions in service. Together with the low flow stresses of the matrices these indicate a marked tendency towards plastic yielding under purely thermal loading. Furthermore, as these materials are intended for applications that involve thermal cycling loads, low cycle fatigue and ratcheting are possible concerns. As a consequence, the thermomechanical behavior of these composites also is an important target for modeling.

It is well known that thermal contact resistances between reinforcements and matrix give rise to a size effect in the overall conductivity of composites, so that even highly conductive particles or fibers fail to increase the overall conductiv-

\footnotetext{
* Corresponding author, Tel.: +43 158801 31712, Fax.: +43 15880131799.

Email address: hjb@ilsb.tuwien.ac.at (Helmut J. Böhm).
} 
ity once their size falls below some critical value, see e.g. [4]. Several analytical approaches to estimating the thermal conduction behavior of composites reinforced with spherical or aligned ellipsoidal particles that have non-ideal interfaces or are coated with interphases can be found in the literature $[4,5,6,7,8,9]$. Diamonds, however, have polyhedral shapes and, in addition, experimental evidence indicates that their $\{100\}$ and $\{111\}$ faces may show different thermal conductances when embedded in a metallic matrix [10]. The above analytical methods do not cover such situations and either semi-analytical models or numerical approaches must be used for studying the thermal conduction behavior of DRMMCs in detail. In the present contribution, a semi-analytical model based on the work of Duschlbauer [11] as well as multi-particle unit cells are employed towards this goal.

Essentially all analytical and most numerical studies of the thermomechanical behavior of particle reinforced composites that are available in the literature have also been based on inhomogeneities of spherical or ellipsoidal shape. Semi-analytical approaches and unit cell models that can account for polyhedral reinforcements in analogy to the models used for thermal conduction are brought to bear on the problem, the interfaces between particles and matrix being assumed to be mechanically perfect.

In the present contribution the main emphasis is put on the development, comparison, and assessment of modeling tools for studying the thermophysical and thermomechanical behavior of ductile matrix composites reinforced by uniformly sized equiaxed polyhedral particles. 


\section{Methods and Modeling Issues}

\subsection{Mori-Tanaka Estimates}

Mean field methods provide relatively simple models for the overall behavior of inhomogeneous materials that can be used for a wide range of reinforcement volume fractions, the fields on the microscale entering via their phase averages. One group of mean field approaches, Mori-Tanaka estimates, approximate the behavior of composites that contain reinforcements at non-dilute volume fractions via dilute inhomogeneities that are subjected to effective matrix fields rather than the macroscopic fields [12]. These effective fields account for the perturbations caused by all other reinforcements in a mean-field sense. Benveniste [13] expressed the central assumption of Mori-Tanaka methods for elastic composites as

$$
\begin{aligned}
\langle\boldsymbol{\varepsilon}\rangle^{(\mathrm{i})} & =\overline{\mathbf{A}}_{\mathrm{dil}}^{(\mathrm{i})}\langle\boldsymbol{\varepsilon}\rangle^{(\mathrm{m})}=\overline{\mathbf{A}}_{\mathrm{dil}}^{(\mathrm{i})} \overline{\mathbf{A}}_{\mathrm{MT}}^{(\mathrm{m})}\langle\boldsymbol{\varepsilon}\rangle \\
\langle\boldsymbol{\sigma}\rangle^{(\mathrm{i})} & =\overline{\mathbf{B}}_{\mathrm{dil}}^{(\mathrm{i})}\langle\boldsymbol{\sigma}\rangle^{(\mathrm{m})}=\overline{\mathbf{B}}_{\mathrm{dil}}^{(\mathrm{i})} \overline{\mathbf{B}}_{\mathrm{MT}}^{(\mathrm{m})}\langle\boldsymbol{\sigma}\rangle,
\end{aligned}
$$

where $\langle\boldsymbol{\varepsilon}\rangle^{(\mathrm{i})},\langle\boldsymbol{\varepsilon}\rangle^{(\mathrm{m})},\langle\boldsymbol{\sigma}\rangle^{(\mathrm{i})}$, and $\langle\boldsymbol{\sigma}\rangle^{(\mathrm{m})}$ are the phase averaged strain and stress tensors of the reinforcement and matrix phases, respectively. $\langle\varepsilon\rangle$ and $\langle\boldsymbol{\sigma}\rangle$ denote the macroscopic strain and stress tensors, $\overline{\mathbf{A}}_{\mathrm{MT}}^{(\mathrm{m})}$ and $\overline{\mathbf{B}}_{\mathrm{MT}}^{(\mathrm{m})}$ the matrix Mori-Tanaka strain and stress concentration tensors, and $\overline{\mathbf{A}}_{\text {dil }}^{(\mathrm{i})}$ and $\overline{\mathbf{B}}_{\text {dil }}^{(\mathrm{i})}$ the dilute strain and stress concentration tensors of the reinforcements. The analogous relationships for the thermal conduction problem take the form

$$
\begin{aligned}
\langle\nabla T\rangle^{(\mathrm{i})} & =\overline{\mathcal{A}}_{\text {dil }}^{(\mathrm{i})}\langle\nabla T\rangle^{(\mathrm{m})}=\overline{\mathcal{A}}_{\mathrm{dil}}^{(\mathrm{i})} \overline{\mathcal{A}}_{\mathrm{MT}}^{(\mathrm{m})}\langle\boldsymbol{\nabla} T\rangle \\
\langle\mathbf{q}\rangle^{(\mathrm{i})} & =\overline{\mathcal{B}}_{\mathrm{dil}}^{(\mathrm{i})}\langle\mathbf{q}\rangle^{(\mathrm{m})}=\overline{\mathcal{B}}_{\text {dil }}^{(\mathrm{i})} \overline{\mathcal{B}}_{\mathrm{MT}}^{(\mathrm{m})}\langle\mathbf{q}\rangle,
\end{aligned}
$$


where $\boldsymbol{\nabla} T$ and $\mathbf{q}$ stand for the thermal gradient and the heat flux vectors, and $\overline{\mathcal{A}}$ and $\overline{\mathcal{B}}$ for the thermal gradient and heat flux concentration tensors. The field and concentration tensors in eqns.(1) are of ranks 2 and 4, respectively, whereas the ranks of the corresponding tensors in eqns.(2) are 1 and 2.

As a consequence of eqn.(2) the Mori-Tanaka gradient concentration tensors of matrix and reinforcements, $\overline{\mathcal{A}}_{\mathrm{MT}}^{(\mathrm{m})}$ and $\overline{\mathcal{A}}_{\mathrm{MT}}^{(\mathrm{i})}$, can be written as [13]

$$
\begin{aligned}
& \overline{\mathcal{A}}_{\mathrm{MT}}^{(\mathrm{m})}=\left((1-\xi) \mathcal{I}+\xi \overline{\mathcal{A}}_{\mathrm{dil}}^{(\mathrm{i})}\right)^{-1} \\
& \overline{\mathcal{A}}_{\mathrm{MT}}^{(\mathrm{i})}=\overline{\mathcal{A}}_{\mathrm{dil}}^{(\mathrm{i})}\left((1-\xi) \mathcal{I}+\xi \overline{\mathcal{A}}_{\mathrm{dil}}^{(\mathrm{i})}\right)^{-1},
\end{aligned}
$$

where $\mathcal{I}$ denotes the rank 2 identity tensor and $\xi$ stands for the reinforcement volume fraction. Analogous expressions hold for the flux, strain and stress concentration tensors. The dilute inclusion gradient concentration tensor, $\overline{\mathcal{A}}_{\mathrm{dil}}^{(\mathrm{i})}$, can in turn be obtained in analogy to Hill's [14] expressions for dilute strain concentration tensors as

$$
\overline{\mathcal{A}}_{\text {dil }}^{(\mathrm{i})}=\left[\mathcal{I}+\mathcal{S} \mathcal{R}^{(\mathrm{m})}\left(\mathcal{K}^{(\mathrm{i})}-\mathcal{K}^{(\mathrm{m})}\right)\right]^{-1}
$$

Here $\mathcal{K}^{(\mathrm{i})}$ is the conductivity tensor of the reinforcements, $\mathcal{K}^{(\mathrm{m})}$ and $\mathcal{R}^{(\mathrm{m})}=$ $\left(\mathcal{K}^{(m)}\right)^{-1}$ are the conductivity and resistivity tensors, respectively, of the matrix, and $\mathcal{S}$ is the Eshelby tensor of the diffusion problem, which is given explicitly e.g. in [15]. The macroscopic (effective) conductivity tensor of the composite, $\mathcal{K}_{\mathrm{MT}}^{*}$, can be estimated from the above expressions as

$$
\mathcal{K}_{\mathrm{MT}}^{*}=\mathcal{K}^{(\mathrm{m})}+\xi\left(\mathcal{K}^{(\mathrm{i})}-\mathcal{K}^{(\mathrm{m})}\right) \overline{\mathcal{A}}_{\mathrm{MT}}^{(\mathrm{i})}
$$

Analogous relations link the inclusion strain concentration tensors $\overline{\mathbf{A}}^{(\mathrm{i})}$, the elasticity tensors $\mathbf{E}^{*}, \mathbf{E}^{(\mathrm{i})}$ and $\mathbf{E}^{(\mathrm{m})}$, the compliance tensor $\mathbf{C}^{(\mathrm{m})}=\left(\mathbf{E}^{(\mathrm{m})}\right)^{-1}$, and the mechanical Eshelby tensor S. The Mori-Tanaka estimates, eqns. (2) 
to (5), hold for general material symmetries of matrix and reinforcements, pertain to aligned ellipsoidal inhomogeneities with perfect interfaces, do not show an intrinsic length scale, and correspond to one of the pertinent HashinShtrikman-type bounds in the case of two-phase materials [16].

The above standard Mori-Tanaka scheme can be extended to handle inhomogeneities of non-ellipsoidal shapes as well as finite interfacial thermal conductances by introducing phase averaged dilute "replacement" inhomogeneity conductivity tensors, $\mathcal{K}^{(\mathrm{i}, \mathrm{r})}$, and the associated dilute "replacement" inhomogeneity gradient concentration tensors, $\overline{\mathcal{A}}_{\text {dil }}^{(\mathrm{i}, \mathrm{r})}$. These replacement tensors must fulfill the consistency condition

$$
\mathcal{K}^{(\mathrm{i}, \mathrm{r})}=\mathcal{K}^{(\mathrm{m})}+\frac{1}{\xi_{\text {dil }}}\left(\mathcal{K}_{\text {dil }}^{*}-\mathcal{K}^{(\mathrm{m})}\right)\left(\overline{\mathcal{A}}_{\text {dil }}^{(\mathrm{i}, \mathrm{r})}\right)^{-1}
$$

which follows from eqn.(5) for the dilute case. Here $\mathcal{K}_{\text {dil }}^{*}$ is the effective conductivity of a composite with dilute reinforcement volume fraction $\xi_{\text {dil }}$. The replacement tensors $\mathcal{K}^{(\mathrm{i}, \mathrm{r})}$ and $\overline{\mathcal{A}}_{\text {dil }}^{(\mathrm{i}, \mathrm{r})}$ are then inserted into eqns.(3) and (5) in lieu of $\mathcal{K}^{(\mathrm{i})}$ and $\overline{\mathcal{A}}_{\text {dil }}^{(\mathrm{i})}$, respectively. This combination of the Mori-Tanaka method with the replacement tensor approach, which was introduced by Duschlbauer [11], is referred to as "RMTM" in the following. When finite interfacial conductances are present between the phases, the replacement tensors depend on the sizes of the reinforcements, so that an absolute length scale is introduced into the Mori-Tanaka scheme. Analogous procedures for the elastic behavior can be defined in terms of replacement elasticity tensors, $\mathbf{E}^{(\mathrm{i}, \mathrm{r})}$, replacement dilute strain concentration tensors, $\overline{\mathbf{A}}_{\text {dil }}^{(\mathrm{i}, \mathrm{r})}$, and dilute effective elasticity tensors, $\mathbf{E}_{\text {dil }}^{*}$, to give

$$
\mathbf{E}^{(\mathrm{i}, \mathrm{r})}=\mathbf{E}^{(\mathrm{m})}+\frac{1}{\xi_{\text {dil }}}\left(\mathbf{E}_{\text {dil }}^{*}-\mathbf{E}^{(\mathrm{m})}\right)\left(\overline{\mathbf{A}}_{\text {dil }}^{(\mathrm{i}, \mathrm{r})}\right)^{-1}
$$


A number of authors modeled mechanically imperfect interfaces by assigning replacement stiffnesses to partially or fully debonded particles, compare e.g. [17]. In the present study, however, only polyhedral particles with mechanically perfect interfaces are considered, for which the replacement tensor approach may be viewed as an alternative to the compliance contribution formalism of Kachanov et al. [18].

For spherical particles of isotropic conductivity that are embedded in an isotropic matrix and show homogeneous interfacial conductances, the replacement tensors can be obtained from well-known scalar relationships and give rise to Mori-Tanaka estimates [5] that are equivalent to the expressions of Hasselman and Johnson [4] as discussed, e.g., in [19]. A similar approach can be followed for ellipsoidal reinforcements with confocally distributed interfacial conductances [11], for which the microfields inside the inhomogeneities maintain the "Eshelby property" of being homogeneous.

If the conductances show more general inhomogeneous distributions over the interface or if the reinforcements are of non-ellipsoidal shape, however, the microfields, the dilute concentration tensors and the Eshelby tensors become position dependent within the inhomogeneities. In such cases numerical methods can be used to advantage for obtaining $\mathcal{K}_{\text {dil }}^{*}, \overline{\mathcal{A}}_{\text {dil }}^{(\mathrm{i}, \mathrm{l})}$ and $/$ or $\mathcal{K}^{(\mathrm{i}, \mathrm{r})}$ as well as their equivalents in elasticity. A very flexible approach to handling these problems uses volume elements made up of a single inhomogeneity of appropriate shape and with appropriately distributed interfacial conductance that is embedded in a large matrix region. In general, these models are subjected to three (in the thermal conduction case) or six (in the elastic case) linearly independent load cases and the microfields are computed numerically, e.g. by the Finite Element method. From these results $\mathcal{K}_{\text {dil }}^{*}$ can be obtained by vol- 
ume averaging over the whole model. For ideal interfaces $\overline{\mathcal{A}}_{\text {dil }}^{(\mathrm{i}, \mathrm{r})}$ can be obtained from volume averages over the inhomogeneity. In cases involving finite interfacial conductances, however, the dilute matrix gradient concentration tensor $\overline{\mathcal{A}}_{\text {dil }}^{(\mathrm{m})}$ is extracted from the numerical solutions and the replacement inclusion concentration tensor is then obtained from the relationship

$$
\overline{\mathcal{A}}_{\text {dil }}^{(\mathrm{i}, \mathrm{r})}=\frac{1}{\xi_{\text {dil }}}\left[\mathcal{I}-\left(1-\xi_{\text {dil }}\right) \overline{\mathcal{A}}_{\text {dil }}^{(\mathrm{m})}\right],
$$

which ensures that interface effects are subsumed into the replacement inhomogeneity. Finally, $\mathcal{K}^{(\mathrm{i}, \mathrm{r})}$ is obtained via eqn. (6). The elastic case can be handled in analogy. In general this procedure provides approximations rather than exact solutions and is not equivalent to the use of phase averaged Eshelby tensors for inhomogeneities with fluctuating microfields as proposed e.g. in [20]. The replacement tensors evaluated with eqns. (6) to (8) were found to be largely independent of the particle volume fraction in the dilute cell, $\xi_{\text {dil }}$. However, some care is required in evaluating these equations because the terms $\mathcal{K}_{\text {dil }}^{*}-\mathcal{K}^{(\mathrm{m})}, \mathbf{E}_{\text {dil }}^{*}-\mathbf{E}^{(\mathrm{m})}$, and $\mathcal{I}-\left(1-\xi_{\text {dil }}\right) \overline{\mathcal{A}}_{\text {dil }}^{(\mathrm{m})}$ vanish for $\xi_{\text {dil }} \rightarrow 0$, making the algorithm potentially sensitive to roundoff errors.

Figure 1 shows a volume element consisting of a single particle with the shape of a regular cubo-octahedron (tetrakaidekahedron) that is embedded in a matrix at a particle volume fraction of $\xi=0.000148$. This is typical of the cells that were subjected to homogeneous gradient boundary conditions to evaluate the replacement tensors. Because both the geometry of the particles and the distribution of the interfacial conductances of configurations of the type displayed in fig. 1 show cubic symmetry, the resulting conductivity tensors, $\mathcal{K}_{\text {dil }}^{*}$ and $\mathcal{K}^{(\mathrm{i}, \mathrm{r})}$, are isotropic, whereas the elasticity tensors, $\mathbf{E}_{\mathrm{dil}}^{*}$ and $\mathbf{E}^{(\mathrm{i}, \mathrm{r})}$, are cubic [21]. When the replacement tensors are combined with an isotropic matrix 
in the Mori-Tanaka formalism, isotropic macroscopic conductivity tensors, $\mathcal{K}_{\mathrm{MT}}^{*}$, and cubic macroscopic elasticity tensors, $\mathbf{E}_{\mathrm{MT}}^{*}$, result because MoriTanaka estimates pertain to aligned reinforcements. A quasi-isotropic overall elastic behavior corresponding to randomly oriented diamond particles can be approximated by "isotropizing" the elasticity tensors, e.g. by using the Hershey-Kröner-Eshelby (HKE) method [22], which was developed to evaluate the macroscopically isotropic elastic response of polycrystals consisting of randomly oriented cubic grains.

\subsection{Multi-Particle Unit Cell Models}

To complement the semi-analytical Mori-Tanaka models presented in section 2.1 multi-particle unit cells were employed for obtaining estimates of the overall thermal and thermomechanical properties of DRMMCs. Such unit cells, which are a well-established micromechanical tool for studying the behavior of discontinuously reinforced composites [23,24,25,26], can provide highly detailed predictions on the microscopic gradient, flux, stress and strain fields in complex microgeometries, but pose much higher requirements in terms of analyst's time and computational power compared to mean field methods.

For the present work unit cells containing 20 randomly positioned particles of equal size and shape were used. To obtain the model microgeometries periodic arrangements of equally sized spheres were generated by the two-step algorithm of Segurado [27], which involves random sequential insertion followed by a Monte-Carlo compaction procedure. Randomly oriented identical regular cubo-octahedra as shown in fig. 1(left) were then inscribed into the spheres, which allowed particle volume fractions of some $34 \%$ to be attained. This 
strategy provides for the generation of unit cells that contain cubo-octahedral particles at different positions and orientations, cubo-octahedra that differ in their orientation only, or spheres that have the same positions and volume fraction as the cubo-octahedra, compare fig. 2. Together with the capability of prescribing different or equal conductances at the $\{100\}$ and $\{111\}$ faces of the diamond particles, the resulting family of models allows various aspects of the phase geometry to be studied in detail at both the micro- and macroscales. The fine Finite Element meshes required for satisfactorily resolving the discontinuities in the interfacial conductance at edges between $\{100\}$ and $\{111\}$ faces led to rather high numbers of degrees of freedom (DOFs) in the thermal conduction models using cubo-octahedral reinforcements, a typical value being 5,160,000. Coarser meshes with approximately 1,300,000 DOFs were used in the thermomechanical studies. Periodicity boundary conditions were applied to all multi-particle unit cells.

In addition to handling the thermal conduction and thermoelastic behaviors of discontinuously reinforced composites, multi-particle unit cell models are well suited to studying their nonlinear thermomechanical responses, such as plastic deformations due to cooling-down processes and other temperature excursions. The main limitation to the models described above lies in the relatively low particle volume fraction that can be attained, which is little more than half of the diamond volume fractions reported for actual samples [28]. Computer generated arrangements of diamond-like particles with volume fractions of up to 64 volume percent were described by Flaquer et al. [29]. These model microgeometries are, however, not periodic and, accordingly, cannot not be studied by the periodic homogenization procedures employed in the present work. 
A further difficulty lies in the limited number of particles that could be handled within the constraints of available computer resources, an issue that is exacerbated by the large number of multi-point constraints required for implementing periodicity boundary conditions, which tend to degrade the efficiency of Finite Element solvers. As discussed in sections 3.1 and 3.2 some anisotropy is present in the overall conductivities and elasticities predicted for the three configurations shown in fig. 2 , which indicates that the unit cells are too small for being proper reference volume elements. Even though ensemble averaging over different configurations can be employed for improving the predictions of the macroscopic properties, unit cells containing a higher number of particles are clearly desirable.

\subsection{Material Parameters}

The same set of thermophysical and thermomechanical constituent material parameters, which correspond to diamond particles and a matrix of pure aluminum, was used for all simulations. Table 1 lists the thermal conductivities, $K$, the isotropic Young's moduli, E, the Poisson numbers, $\nu$, and the bulk moduli, $B$, used for the constituents. The conductances for the $\{100\}$ and $\{111\}$ faces of synthetic diamond particles embedded in aluminum given in table 2 are preliminary estimates that aim at accounting for differences in the behavior of the two sets of faces, the lower reactivity of the $\{111\}$ faces leading to reduced bonding and to a lower interfacial conductance [30].

Whereas temperature independent elastic constants were employed in all studies of the thermomechanical behavior of DRMMCs, temperature dependent hardening curves were specified following the results given for aluminum 99.99 
by Chinh et al. [31]. Table 3 lists the corresponding initial yield stresses, $\sigma_{\mathrm{y}, 0}$, for a number of temperatures. The coefficients of thermal expansion of the constituents were prescribed as functions of temperature following [32], see table 4 .

\section{Results and Discussion}

All unit cells discussed in this section were meshed with the preprocessor HyperMesh (Altair Computing, Troy, MI) and the analysis runs were carried out with the general purpose Finite Element code ABAQUS/Standard (Simulia, Providence, RI). All models were meshed with 10-node tetrahedral elements.

The multi-particle unit cells were generated such that all particles are of equal size. Even though Mori-Tanaka methods do not describe uniformly sized particles, a well-defined absolute length scale is introduced into thermal conduction models by the choice of the interfacial conductance $h$. In the following the sizes of cubo-octahedral particles are given in terms of the diameter of the circumscribed sphere, $d$. The diameters of spherical particles, $d_{\mathrm{s}}$, were chosen to obtain the same volume as the diamond particles, which implies $d_{\mathrm{s}}=\frac{2}{\sqrt{5}}\left(\frac{3}{\pi}\right)^{1 / 3} d$. For most of the studies presented in the following the size of cubo-octahedral particles was chosen as $d=200 \mu \mathrm{m}$, so that the diameter of the equivalent spheres results as $d_{\mathrm{s}}=176 \mu \mathrm{m}$.

For a given particle size an equivalent homogeneous interfacial conductance $h_{\text {eqv }}$ can be defined by requiring that it gives rise to the same replacement conductivity $\mathcal{K}^{(\mathrm{i}, \mathrm{r})}$ as the inhomogeneous interfacial conductances listed in table 2. For cubo-octahedra of $d=200 \mu \mathrm{m}$ this equivalent homogeneous interfacial 
conductance, $h_{\text {eqv }, 200}$, takes a value of some $27.7 \mathrm{MW} / \mathrm{Km}^{2}$.

\subsection{Thermal Conduction Behavior}

In fig. 3 predictions for the effective thermal conductivity, $K^{*}$, of a DRMMC with a diamond volume fraction of $\xi=0.34$ are compared for different interfacial conductance scenarios and different micromechanical models. For ideal interfaces, $h \rightarrow \infty$, marked as "MTM, perfect", and for vanishing interfacial conductances, $h \rightarrow 0$, marked as "MTM, voids", the effective conductivity can be computed by standard Mori-Tanaka methods and does not depend on the particle size. Semi-analytical Mori-Tanaka predictions are shown for cubooctahedral diamond particles with homogeneous ("RMTM, hom") and inhomogeneous ("RMTM, inh") interfacial conductances, where the former use $h_{\text {eqv } 200}$. Both curves show the sigmoid dependence of $K^{*}$ on the particle size known from the Hasselman--Johnson model [4]. Some effects of the interfacial scenarios can be seen in the size range $1 \mu \mathrm{m} \leq d \leq 100 \mu \mathrm{m}$, where the inhomogeneous conductances lead to a slightly higher macroscopic conductivity than the homogeneous ones. The latter do not lie within the three-point bounds of Torquato and Rintoul [8] (evaluated for $h_{\text {eqv,200 }}$ and non-interpenetrating spheres of equal size, and marked as "3PB, hom") for part of the size range shown, which is not surprising because they describe physically different scenarios. At the critical diameter of $d_{\mathrm{s}, \mathrm{cr}}=19.7 \mu \mathrm{m}$, where the effective conductivity of the sphere-reinforced composite equals that of the matrix, the upper and lower three-points bounds coincide. Effective conductivities obtained with unit cell UCDA for ideal, homogeneous and inhomogeneous interfacial conductances at $d=200 \mu \mathrm{m}$ ("UCDA, inh") slightly exceed the Mori-Tanaka data. 
The replacement conductivities evaluated for spherical and cubo-octahedral inhomogeneities and different interfacial scenarios are listed in table 5. For perfect interfaces the replacement conductivity of the cubo-octahedra coincided with the physical conductivity of diamonds. As intended, the replacement conductivities of cubo-octahedra with inhomogeneous and equivalent homogeneous interfacial conductances are nearly equal. However, those of spheres with the same volume and the same interfacial conductance are about $3 \%$ smaller. The corresponding predictions for the effective conductivities, 399.7 $\mathrm{W} / \mathrm{Km}$ and $392.1 \mathrm{~W} / \mathrm{Km}$ (compare table 6 ) differ by about $1.8 \%$.

Table 6 lists predictions for spherical and cubo-octahedral particles at a volume fraction of $\xi=0.34$ that were obtained with a number of models and interfacial scenarios. For the the considered particle size of $200 \mu \mathrm{m}$ about $72 \%$ of the potential improvement in conductivity that can be achieved by 34 vol.\% of diamonds is attained. The results obtained from unit cells with spherical particles, $K_{\mathrm{UCS}}^{*}$, approach the predictions of the Hasselman-Johnson model [4], which coincides with the Mori-Tanaka model for spherical particles. Compared to the spheres, cubo-octahedral particles with homogeneous interfacial conductances of $h_{\text {eqv } 200}$ give rise to macroscopic conductivities that are about $1.5 \%$ higher in the Mori-Tanaka approximation $\left(K_{\mathrm{MTM}}^{*}\right)$ and about $2 \%$ higher in the unit cell models ( $K_{\mathrm{UCDA}}^{*}$ and $K_{\mathrm{UCDB}}^{*}$ ). If inhomogeneous interfacial conductances are introduced into the unit cell models there is an additional minor increase in the macroscopic conductivity. The differences between the predictions obtained with the two unit cells UCA and UCB, however, are very small.

The symmetry of the effective conductivities predicted by the unit cells is probed in table 7, where results obtained for temperature gradients in the $x$-, $y$ - 
and $z$-directions are compared for three configurations. The largest differences are found for the scenario of perfect interfaces, for which the conductivity contrast is highest. The predictions obtained for cells UCDA and UCDB differ only by a small amount, indicating that the positions of the particle centers rather than the orientations of the cubo-octrahedra control the macroscopic anisotropy. In all cases considered the differences in $K^{*}$ are less than $1 \%$ and the averaged conductivities $K_{\text {avg }}^{*}$ can be considered as valid estimates.

Figures 4 and 5 concentrate on the distribution of microscopic heat fluxes in the particles. In fig.4 the three unit cells shown in fig.2 are combined with homogeneous interfacial conductances, $h_{\text {eqv }, 200}$, and subjected to unit thermal gradients acting in $z$-direction. The resulting intra-particle heat flux distributions in the individual inhomogeneities are represented in terms of averages and standard deviations. The spherical particles consistently show slightly smaller averages and significantly smaller standard deviations of the fluxed than the cubo-octahedra. This behavior is related to the fact that in dilute spheres the microfields are homogeneous, whereas in dilute polyhedra they are not. In fig.5 the fluxes in the particles are compared for unit cells UCDA and UCDB, which differ only in the orientations of the cubo-octahedra. Even though inhomogeneous interfacial conductances were prescribed, the two unit cells show only minor differences in the averages and standard deviations of the intra-particle distributions of the fluxes. In both cases the fluxes in given particles are mainly governed by the positions of the centers, which is evident in the nearly identical variations of the average fluxes with the particle numbers.

Taken together, the above results allow the conclusion that for moderate particle volume fractions the combination of the Mori-Tanaka method with replace- 
ment tensors obtained from numerical models provides valid predictions for the overall conductivity of composites reinforced by cubo-octahedral particles with inhomogeneous interfacial conductances. For an elevated particle volume fraction of $\xi=0.6$ and a particle size of $d=200 \mu \mathrm{m}$ the semi-analytical MoriTanaka method predicts a macroscopic conductivity of $K_{\mathrm{MTM}}^{*}=596 \mathrm{~W} / \mathrm{Km}$, which falls within the three-point bounds of $K_{\mathrm{LB}}^{*}=567 \mathrm{~W} / \mathrm{Km}$ and $K_{\mathrm{UB}}^{*}=639$ $\mathrm{W} / \mathrm{Km}$ evaluated for non-interpenetrating spherical particles having an interfacial conductance of $h_{\text {eqv }, 200}$. All of the above values lie below the maximum

experimentally determined conductivity of $K_{\mathrm{exp}, \max }^{*} \approx 670 \mathrm{~W} / \mathrm{Km}$ reported for Al-matrix DRMMCs with particle sizes of $d \approx 100 \mu \mathrm{m}$ and particle volume fractions of some $60 \%$ that were produced by gas pressure infiltration $[10,30]$. This indicates that at least in some actual materials the interfacial conductances are considerably higher than assumed in the present study. In addition, the tendency of Mori-Tanaka methods to underestimate the overall conductivities, especially at elevated particle volume fractions, may play some role. Finally, the predictions of the unit cell models provide clear evidence that the inhomogeneous distributions of the interfacial conductances on the particles' faces have only a very limited influence on the overall conductivity of the composite and on the phase averages of the microfields.

\subsection{Thermomechanical Behavior}

Predictions for the macroscopic elastic moduli and coefficients of thermal expansion for a particle volume fraction $\xi=0.34$ at room temperature are collected in table 8. Here "MTM, sph" stands for the classical Mori-Tanaka estimates employing spherical particles [13] and "RMTM, diam" for the Mori- 
Tanaka method using replacement tensors evaluated for cubo-octahedral particles. For the latter case isotropic effective elasticity tensors were obtained by applying the HKE method. Torquato's three-point estimates [33] evaluated for spherical particles of uniform size are listed under the heading "3PE, sph", and results from unit cell models shown in fig. 2 are marked as "UCS" and "UCDA". Because the unit cells were too small to be reference volume elements some anisotropy was present in their responses, the differences between diagonal terms of the elasticity tensor being less than $1.5 \%$, however. The unit cell results listed in table 8 pertain to macroscopic elasticity tensors made quasi-isotropic by the HKE algorithm. In contrast to the effective thermal conductivities discussed in section 3.1 the predicted elastic tensors do not have an intrinsic length scale.

Both the Mori-Tanaka methods and the multi-particle unit cells predict a stiffer macroscopic behavior for cubo-octahedral particles compared to spheres, the effect being more pronounced in the unit cell models. The overall elastic behavior obtained from the Mori-Tanaka methods is more compliant than the predictions of the three-point estimates and the unit cells. This is due to the well-known fact that Mori-Tanaka approaches provide lower estimates for the stiffness of composites in which the reinforcements are stiffer than the matrix; for the spherical particles the MTM results correspond to the Hashin-Shtrikman lower bounds. For a particle volume fraction of $\xi=0.6$ the predictions for the Young's moduli and the Poisson number are $E^{*}=228.8$ GPa and $\nu^{*}=0.262$ for spherical particles and $E^{*}=255.9 \mathrm{GPa}$ and $\nu^{*}=0.241$ for cubo-octahedra, respectively. Interestingly, even though a stiffer overall behavior is obtained with cubo-octahedral particles, both their replacement Young's modulus, $E^{(\mathrm{i}, \mathrm{r})}=1001.3 \mathrm{GPa}$, and their replacement bulk modulus, 
$B^{(\mathrm{i}, \mathrm{r})}=395.5 \mathrm{GPa}$, are lower than the corresponding physical moduli listed in table 1. Evidently, the interplay between $\mathbf{E}^{(\mathrm{i}, \mathrm{r})}$ and $\overline{\mathbf{A}}_{\text {dil }}^{(\mathrm{i}, \mathrm{r})}$ is non-trivial.

Inspection of the results of the numerical models using cubo-octahedral particles indicated that at the interfaces singularities may be present in the elastic stress and strain fields in the matrix at reentrant edges and corners. Even though such singularities cannot be resolved with the Finite Elements models employed in the present study, their presence does not compromise the evaluation of the reduced elastic concentration tensors, for which the volume averaged strains in the inhomogeneities are required. They lead to difficulties, however, in using criteria for macroscopic yielding that are based on the maximum of the equivalent stress in the matrix as predicted by multi-particle unit cells.

Due to the marked thermal expansion contrast between the constituents and the low initial yield stress of the matrix, compare tables 3 and 4, a strong tendency of DRMMCs toward matrix yielding under thermal loading must be expected. Plastic yielding of macroscopically isotropic composites under pure thermal loading cannot be described by Mori-Tanaka methods, which always predict hydrostatic matrix stress states under such conditions. Unit cell methods, however, are well suited to the task. Thermoelastic modeling with unit cell UCS (20 spherical particles) indicated that the temperature change required for initiating local plastic yielding is more than an order of magnitude smaller in a DRMMC with a matrix of Al99.99 compared to a high strength aluminum alloy reinforced by $\mathrm{SiC}$ particles of the same volume fraction.

Thermoelastoplastic analysis employing the multi-particle unit cells shown in 
fig. 2 and the temperature dependent constituent properties listed in tables 3 and 4 can be used to study the macroscopic thermomechanical responses as well as the effects of local thermal stresses and strains in DRMMCs. Figure 6 (left) shows the distribution of the accumulated equivalent plastic strains, $\bar{\varepsilon}_{\text {eqv,pl }}^{(\mathrm{m})}$, predicted for the matrix domain of unit cell UCDA after cooling down from a stress-free temperature of $450 \mathrm{~K}$ to room temperature. The plastic strains can be seen to be highly inhomogeneous and to exceed a value of 0.01 over a considerable part of the volume element. Because hardly any elastic matrix regions remain, the matrix may be viewed as fully yielded in this state. Heating up to $393 \mathrm{~K}$ from this state leads to further plastic yielding as displayed in fig. 6 (right), where especially the increased extent of regions with accumulated equivalent plastic strains in excess of 0.1 is noteworthy. To further illustrate this behavior, the evolution of the equivalent accumulated plastic strain in the matrix is shown in fig. 7, where the phase averages and standard deviations of $\bar{\varepsilon}_{\text {eqv,pl }}^{(\mathrm{m})}$ are plotted for selected temperatures during the heating-up process. Temperature changes of up to $20 \mathrm{~K}$ give rise to some local yielding, but have little influence on the distribution of the plastic strains in the matrix. Temperature excursions in excess of $40 \mathrm{~K}$, however, lead to an increase in the averages of $\bar{\varepsilon}_{\mathrm{eqv}, \mathrm{pl}}^{(\mathrm{m})}$, which indicates to bulk yielding of the matrix, and to a marked rise in the standard deviations, the latter indicating an intensifying inhomogeneity of the plastic strains.

Figure 8 compares the macroscopic responses of initially stress-free (virgin) and cooled-down diamond-aluminum composites to uniaxial tensile loading as predicted with unit cell UCDA. Up to an applied stress of approximately $20 \mathrm{MPa}$ there is little difference between the two curves, but for tensile stresses exceeding $25 \mathrm{MPa}$ the residual stresses in the cooled-down material lead to a 
markedly weaker hardening behavior.

The above modeling results clearly indicate that DRMMCs tend to be subject to marked matrix plasticity effects under thermal loading. The resulting residual stress states may also strongly influence the mechanical responses. This behavior must be accounted for in the development, production and use of such materials for heat sink applications.

\section{Conclusions}

The thermophysical and thermomechanical behavior of metal matrix composites reinforced by polyhedral particles was studied by a Mori-Tanaka method employing replacement conductivity, elasticity and concentration tensors as well as by multi-particle unit cells. The material constituent parameters were chosen to correspond to a matrix of pure aluminum reinforced by diamond particles, and homogeneous as well as inhomogeneous distributions of the interfacial conductances were investigated.

The predictions of the two modeling approaches were compared for a moderate particle volume fraction of $\xi=0.34$ and found to be in good agreement. On this basis the respective strengths of the two classes of models were made use of by studying the thermal and thermoelastic responses at elevated diamond volume fractions with the Mori-Tanaka method and by using the unit cells for predicting the thermoelastoplastic behavior as well as for focusing in on details of the fields on the microscale.

The replacement tensor approach used in this study may also be combined with other mean field methods that can be formulated in terms of concentra- 
tion tensors, such as classical self-consistent models and differential schemes.

The results of the modeling effort support the viability of the concept of using DRMMCs as high-conductivity heat sink materials. The main hurdles that have to be overcome are, on the one hand, the provision of interfaces of high thermal conductance which allow overall thermal conductivities in excess of $500 \mathrm{~W} / \mathrm{Km}$ to be obtained from reasonably small diamond particles. On the other hand, a high mechanical quality of the interfaces and a void-free matrix must be reliably achieved in order to allow the composite to survive the high thermal stresses inherent in the given combination of constituents. Also, service conditions may have to be chosen to minimize cyclic plastic yielding due to temperature excursions.

\section{Acknowledgments}

This work was carried out within the framework of the Integrated European Project ExtreMat (contract NMP-CT-2004-500253) with financial support from the European Community. It only reflects the views of the authors and the European Community is not liable from any use of the information contained therein.

\section{References}

[1] B. Maruyama, R.K. Everett, A. Morrish, P. Natishan, A.E. Edelstein, L.S. Cook. Diamond reinforced composites. In: A.L. Bunsell, J.F. Jamet, A. Massiah editors, ECCM5, Developments in the Science and Technology of Composite Materials, Bordeaux (1992), pp. 715-720. 
[2] C. Owers. Industrial diamond: applications, economics and a view to the future. Indust. Diam. Rev. 60 (2000) 176-177.

[3] T.W. Clyne. Thermal and electrical conduction in MMCs. In: A. Kelly, C. Zweben editors. Comprehensive Composite Materials. Pergamon Press, Oxford (2000), pp. 447-468.

[4] D. Hasselman, L. Johnson. Effective thermal conductivity of composites with interfacial thermal barrier resistance. J. Compos. Mater. 21 (1987) 508-515.

[5] Y. Benveniste. On the effective thermal conductivity of multiphase composites. J. Appl. Math. Phys. 37 (1986) 696-713.

[6] Y. Benveniste, T. Miloh. On the effective thermal conductivity of coated shortfiber composites. J. Appl. Phys. 69 (1991) 1337-1344.

[7] M. L. Dunn, M. Taya. The effective thermal conductivity of composites with coated reinforcement and the application to imperfect interfaces. J. Apply. Phys. 77 (1995) 4954-4960.

[8] S. Torquato, D.M. Rintoul. Effect of the interface on the properties of composite media. Phys. Rev. Lett. 75 (1995) 4067-4070.

[9] C.W. Nan, G. Liu, Y.H. Lin, M. Li. Interface effects on thermal conductivity of carbon nanotube composites. Appl. Phys. Lett. 85 (2004) 3549-3551.

[10] S. Kleiner, F. Khalid, P. Ruch, S. Meier, O. Beffort. Effect of diamond crystallographic orientation on dissolution and carbide formation in contact with liquid aluminium, Scr. mater. 55 (2006) 291-294.

[11] D. Duschlbauer. Computational Simulation of the Thermal Conductivity of MMCs under Consideration of the Inclusion-Matrix Interface, VDI-Verlag, Düsseldorf, Germany, 2004. 
[12] T. Mori, K. Tanaka. Average stress in the matrix and average elastic energy of materials with misfitting inclusions. Acta Metall. 21 (1973) 571-574.

[13] Y. Benveniste. A new approach to the application of Mori-Tanaka's theory in composite materials. Mech. Mater. 6 (1987) 147-157.

[14] R. Hill, A self-consistent mechanics of composite materials, J. Mech. Phys. Sol. 13 (1965) 213-222.

[15] H. Hatta, M. Taya, Equivalent inclusion method for steady state heat conduction in composites, Int. J. Engng. Sci. 24 (1986) 1159-1172.

[16] G.J. Weng. The theoretical connection between Mori-Tanaka theory and the Hashin-Shtrikman-Walpole bounds. Int. J. Engng. Sci. 28 (1990) 1111-1120.

[17] K. Tohgo, G.J. Weng. A progressive damage mechanics in particle-reinforced metal-matrix composites under high triaxial tension; J. Engng. Mater. Technol. 116 (1994) 414-420.

[18] M. Kachanov, I. Tsukrov, B. Shafiro. Effective moduli of solids with cavities of various shapes. Appl. Mech. Rev. 47 (1994) S151-S174.

[19] H.J. Böhm, S. Nogales. Mori-Tanaka models for the thermal conductivity of composites with interfacial resistance and particle size distributions. Compos. Sci. Technol., in print (2007), doi:10.1016/j.compscitech.2007.06.009

[20] H. Nozaki, M. Taya. Elastic fields in a polyhedral inclusion with uniform eigenstrains and related problems. J. Appl. Mech. 68 (2001) 441-452.

[21] J.F. Nye. Physical Properties of Crystals. Oxford University Press, Oxford (1985).

[22] H. Ledbetter. Monocrystal-polycrystal elastic constants of a stainless steel. Phys. Stat. Sol. A 85 (1984) 89-96. 
[23] A.A. Gusev. Representative volume element size for elastic composites: A numerical study. J. Mech. Phys. Sol. 45 (1997) 1449-1459.

[24] H.J. Böhm, W. Han. Comparisons between three-dimensional and twodimensional multi-particle unit cell models for particle reinforced metal matrix composites. Modell. Simul. Mater. Sci. Engng. 9 (2001) 47-65.

[25] J. Segurado, C. González, J. LLorca. A numerical investigation of the effect of particle clustering on the mechanical properties of composites. Acta mater. 51 (2003) 2355-2369.

[26] D. Duschlbauer, H.J. Böhm, H.E. Pettermann. Computational simulation of composites reinforced by planar random fibers: Homogenization and localization by unit cell and mean field approaches. J. Compos. Mater. 40 (2006) 2217-2234.

[27] J. Segurado. Micromecánica computacional de materiales compuestos reforzados con partículas. Doctoral Thesis, Universidad Politécnica de Madrid (2004).

[28] J. Molina, E. Piñero, J. Narciso, C. García-Cordovilla, E. Louis, Liquid metal infiltration into ceramic particle preforms with bimodal size distributions, Curr. Opin. Sol. State Mater. Sci. 9 (2006) 202-210.

[29] J. Flaquer, A. Ríos, A. Martín-Meizoso, S. Nogales, H.J. Böhm. Effect of diamond shapes and associated thermal boundary resistance on thermal conductivity of diamond-base composites. Comput. Mater. Sci. 49 (2007) 156163.

[30] P.W. Ruch, O. Beffort, S. Kleiner, L. Weber, P.J. Uggowitzer. Selective interfacial bonding in $\mathrm{Al}(\mathrm{Si})$-diamond composites and its effect on thermal conductivity. Compos. Sci. Technol. 66 (2006) 2677-2685.

[31] N.Q. Chinh, J. Illy, Z. Horita, T.G. Langdon. Using the stress-strain relationships to propose regions of low and high temperature plastic deformation 
in aluminum. Mater. Sci. Engng. A 410-411 (2005) 234-238.

[32] Y.S. Touloukian, R.K. Kirby, R.E. Taylor, T.Y.R. Lee (Eds). Thermal Expansion in Metallic Elements and Alloys. IFI/Plenum, New York (1975).

[33] S. Torquato. Morphology and effective properties of disordered heterogeneous media. Int. J. Sol. Struct. 35 (1998) 2385-2406. 


\section{Tables}

Table 1

Thermal and elastic material parameters of the constituents

\begin{tabular}{|l|c|c|c|c|}
\hline & $K[\mathrm{~W} / \mathrm{Km}]$ & $E[\mathrm{GPa}]$ & $\nu[]$ & $B[\mathrm{GPa}]$ \\
\hline Diamond & 1800 & 1050 & 0.1 & 438 \\
\hline Aluminum & 237 & 70 & 0.33 & 69 \\
\hline
\end{tabular}

Table 2

Estimates for the thermal conductances of the diamond-aluminum interfaces

\begin{tabular}{|c|c|}
\hline$h_{\{100\}}\left[\mathrm{MW} / \mathrm{Km}^{2}\right]$ & $h_{\{111\}}\left[\mathrm{MW} / \mathrm{Km}^{2}\right]$ \\
\hline 100 & 20 \\
\hline
\end{tabular}

Table 3

Initial yield stress $\sigma_{\mathrm{y}, 0}$ of $\mathrm{Al} 99.99$ as function of the temperature $T$ (data estimated from curves given in [31]).

\begin{tabular}{|c|c|c|c|c|c|c|}
\hline & $T=293 \mathrm{~K}$ & $T=353 \mathrm{~K}$ & $T=433 \mathrm{~K}$ & $T=623 \mathrm{~K}$ & $T=673 \mathrm{~K}$ & $T=738 \mathrm{~K}$ \\
\hline$\sigma_{\mathrm{y}, 0}[\mathrm{MPa}]$ & 20.8 & 17.5 & 12.0 & 7.5 & 5.8 & 4.3 \\
\hline
\end{tabular}


Table 4

Coefficients of thermal expansion of aluminum and diamonds as functions of the temperature $T[32]$

\begin{tabular}{|c|c|c|}
\hline$T[\mathrm{~K}]$ & $\alpha_{\mathrm{Al}}[1 / \mathrm{K}]$ & $\alpha_{\text {diam }}[1 / \mathrm{K}]$ \\
\hline 200 & $20.3 \times 10^{-6}$ & $1.50 \times 10^{-6}$ \\
\hline 300 & $23.1 \times 10^{-6}$ & $1.50 \times 10^{-6}$ \\
\hline 400 & $25.1 \times 10^{-6}$ & $1.79 \times 10^{-6}$ \\
\hline 500 & $26.4 \times 10^{-6}$ & $2.70 \times 10^{-6}$ \\
\hline 600 & $28.4 \times 10^{-6}$ & $3.17 \times 10^{-6}$ \\
\hline 800 & $34.0 \times 10^{-6}$ & $3.81 \times 10^{-6}$ \\
\hline
\end{tabular}

Table 5

Replacement conductivities of diamond particles embedded in an aluminum matrix for different interfacial scenarios and particle diameters of $d=200 \mu \mathrm{m}$ (diamonds) and $d_{\mathrm{S}}=176 \mu \mathrm{m}$ (spheres).

\begin{tabular}{|c|c|c|c|c|c|}
\hline & sph, perfect & sph, hom & diam, perfect & diam, hom & diam, inh \\
\hline$K^{(\mathrm{i}, \mathrm{r})}[\mathrm{W} / \mathrm{Km}]$ & 1800.0 & 1035.4 & 1800.0 & 1067.2 & 1067.4 \\
\hline
\end{tabular}


Table 6

Comparison of effective conductivities $K^{*}$ predicted by the unit cells UCDA, UCDB and UCS (see fig.2), by the semi-analytical Mori-Tanaka model (RMTM, cubooctahedral particles), and by the Hasselman-Johnson model (HJ, spherical particles) for different interfacial conductance scenarios for a diamond volume fraction of $\xi=0.34$ and particle diameters of $d=200 \mu \mathrm{m}$ (diamonds) and $d_{\mathrm{s}}=176 \mu \mathrm{m}$ (spheres).

\begin{tabular}{|l|c|c|c|c|c|}
\hline & $K_{\mathrm{UCDA}}^{*}[\mathrm{~W} / \mathrm{Km}]$ & $K_{\mathrm{UCDB}}^{*}[\mathrm{~W} / \mathrm{Km}]$ & $K_{\mathrm{RMTM}}^{*}[\mathrm{~W} / \mathrm{Km}]$ & $K_{\mathrm{UCS}}^{*}[\mathrm{~W} / \mathrm{Km}]$ & $K_{\mathrm{HJ}}^{*}[\mathrm{~W} / \mathrm{Km}]$ \\
\hline perfect & 469.0 & 469.8 & 461.4 & 457.7 & 453.0 \\
\hline hom & 404.0 & 404.2 & 399.7 & 393.7 & 392.1 \\
\hline inh & 405.1 & 405.2 & 401.0 & - & - \\
\hline
\end{tabular}


Table 7

Effective conductivities obtained by applying thermal gradients in $x-, y-$ and $z$ directions to the unit cells UCDA, UCDB, and UCS (see fig.2) for different conductance scenarios, a diamond volume fraction of $\xi=0.34$, and particle diameters of $d=200 \mu \mathrm{m}$ (diamonds) and $d_{s}=176 \mu \mathrm{m}$ (spheres).

\begin{tabular}{|l|l|c|c|c|c|}
\hline & & $K_{x}^{*}[\mathrm{~W} / \mathrm{Km}]$ & $K_{y}^{*}[\mathrm{~W} / \mathrm{Km}]$ & $K_{z}^{*}[\mathrm{~W} / \mathrm{Km}]$ & $K_{\text {avg }}^{*}[\mathrm{~W} / \mathrm{Km}]$ \\
\hline UCDA & perfect & 471.9 & 467.3 & 467.9 & 469.0 \\
\hline & hom & 405.4 & 403.0 & 403.4 & 404.0 \\
\hline & inh & 406.5 & 404.1 & 404.6 & 405.1 \\
\hline UCDB & perfect & 472.7 & 468.7 & 467.9 & 469.8 \\
\hline & hom & 405.7 & 403.6 & 403.3 & 404.2 \\
\hline & inh & 406.7 & 404.8 & 404.2 & 405.2 \\
\hline UCS & perfect & 459.8 & 457.2 & 456.0 & 457.7 \\
\hline & hom & 394.7 & 393.5 & 393.0 & 393.7 \\
\hline
\end{tabular}


Table 8

Predictions for the effective elastic moduli of diamond reinforced aluminum at a diamond volume fraction of $\xi=0.34$ obtained by Mori-Tanaka methods (MTM and RMTM), Torquato's three-point estimates (3PE), and unit cells UCS and UCDA.

\begin{tabular}{|l|l|c|c|}
\hline & $E^{*}[\mathrm{GPa}]$ & $G^{*}[\mathrm{GPa}]$ & $B^{*}[\mathrm{GPa}]$ \\
\hline MTM, sph & 131.2 & 50.7 & 106.1 \\
\hline RMTM, diam & 133.9 & 51.9 & 106.7 \\
\hline 3PE, sph & 137.3 & 53.3 & 107.3 \\
\hline UCS & 135.8 & 52.7 & 106.6 \\
\hline UCDA & 141.7 & 55.3 & 108.0 \\
\hline
\end{tabular}




\section{Figures}
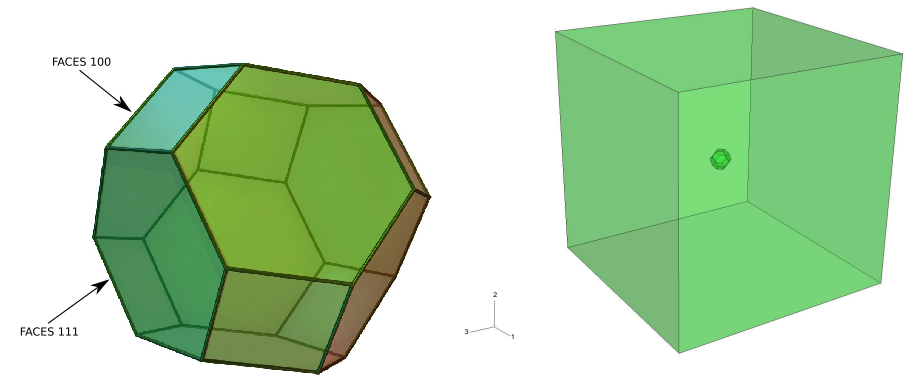

Fig. 1. Cubo-octahedral geometry of the diamond particles used in all numerical models (left) and dilute unit cell used for obtaining the thermal replacement concentration and conduction tensors (right)
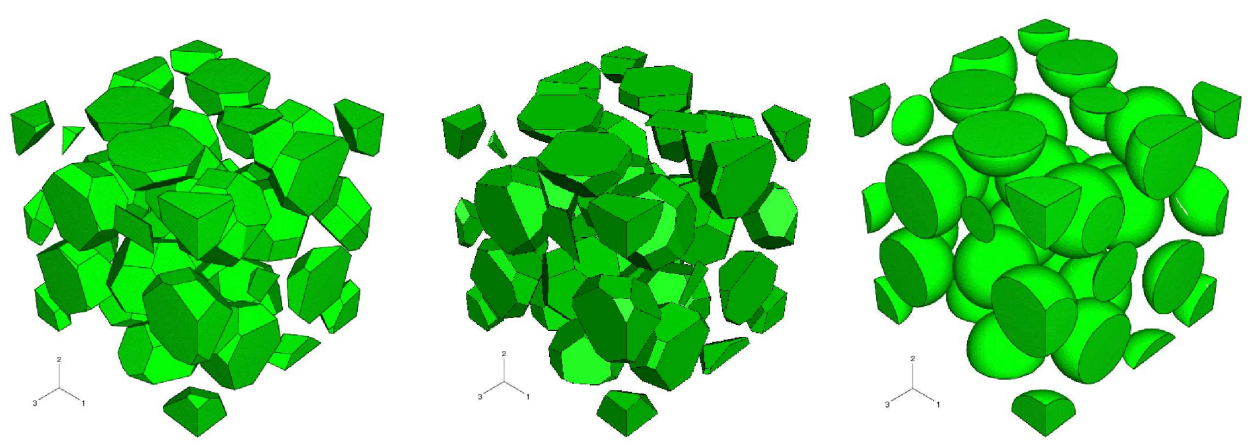

Fig. 2. Multi-particle unit cells, UCDA (left), UCDB (middle) and UCS (right), used in the analysis. The three unit cells have the same reinforcement volume fraction of $\xi=0.34$ and use the same particle centers. 


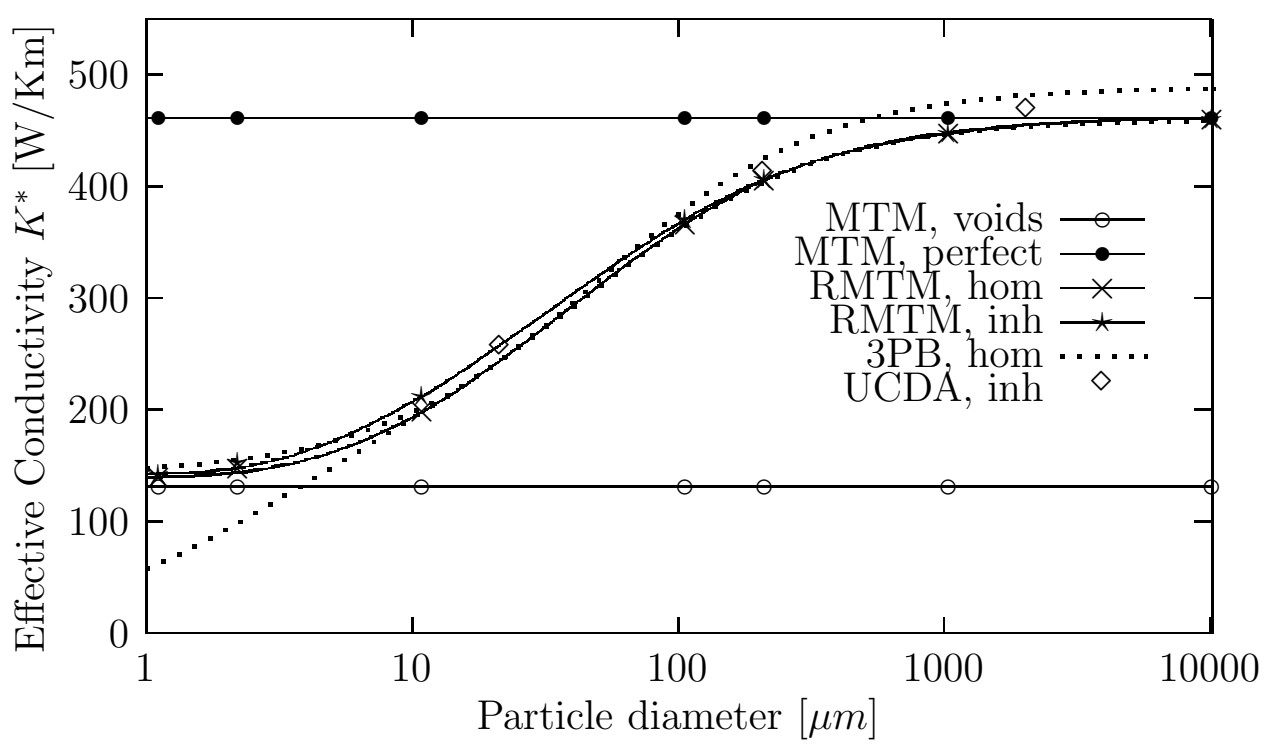

Fig. 3. Predictions for the effective conductivity of aluminum reinforced with uniformly sized diamonds at $\xi=0.34$ obtained with Mori-Tanaka methods for four different interfacial conductance scenarios as functions of the particle size. "MTM, perfect" represents perfect interfacial conductances, "MTM, voids" vanishing interfacial conductances, "RMTM, hom" a homogeneous conductance on all faces $\left(h_{\text {eqv }, 200}=27.7 \mathrm{MW} / \mathrm{Km}^{2}\right.$ for $\left.d=200 \mu \mathrm{m}\right)$, and "RMTM, inh" different conductances for the $\{100\}$ and $\{111\}$ faces. For comparison the three-point bounds of Torquato and Rintoul [8] pertaining to spheres with homogeneous interfacial conductances ("3PB, hom") as well as unit cell results ("UCDA") are plotted. 


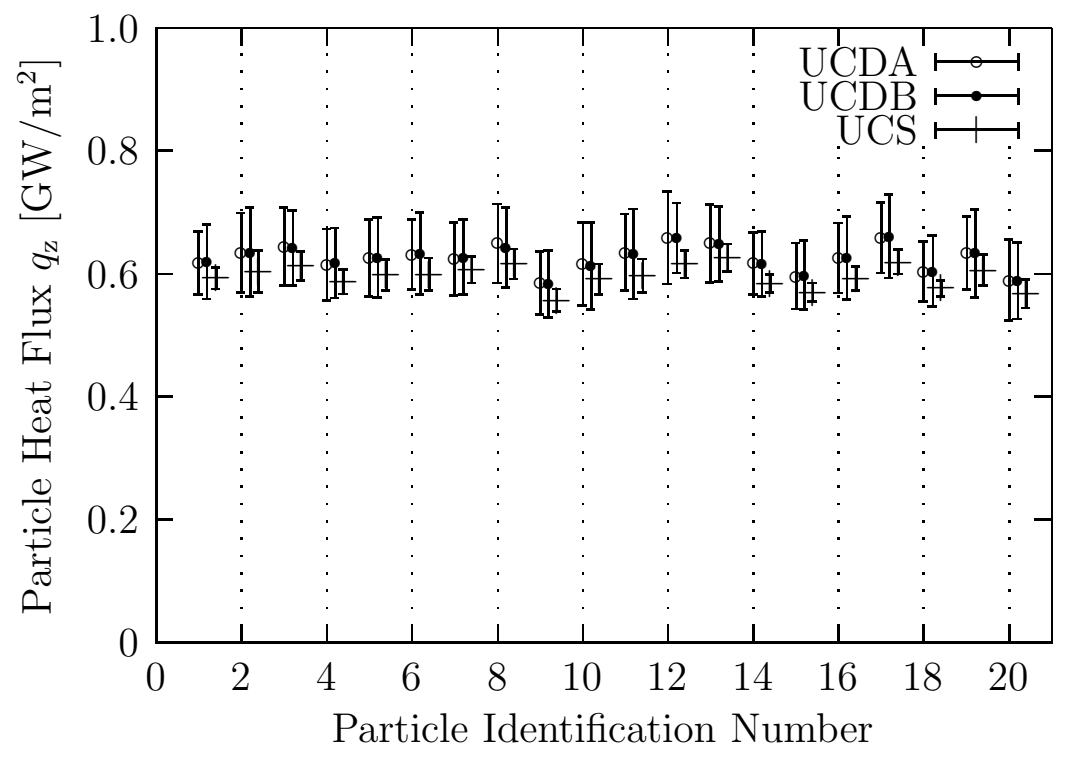

Fig. 4. Heat fluxes in the particles (means and standard deviations) predicted by unit cells UCDA, UCDB and UCS (compare fig.2) for a homogeneous interfacial conductance of $h_{\text {eqv }, 200}=27.7 \mathrm{MW} / \mathrm{Km}^{2}$ and loading by a unit thermal gradient acting in $z$-direction (particle diameters $d=200 \mu \mathrm{m}$ for the diamonds and $d_{s}=176$ $\mu \mathrm{m}$ for the spheres, $\xi=0.34)$. 


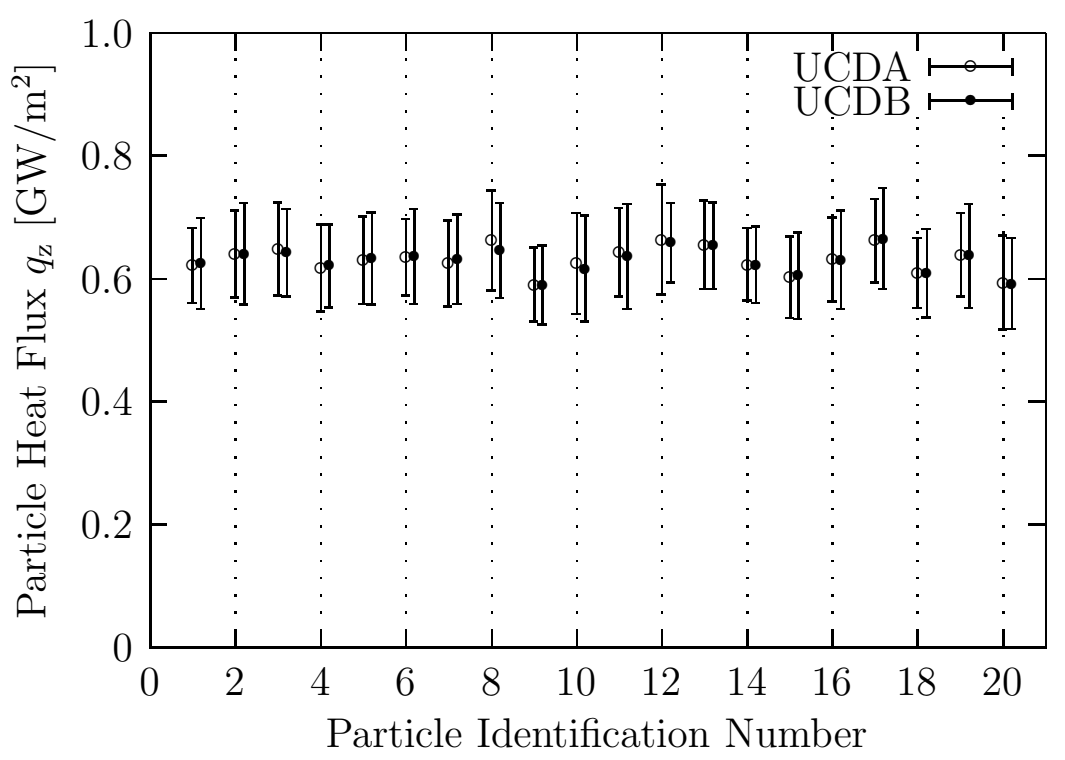

Fig. 5. Heat fluxes in the particles (means and standard deviations) predicted by unit cells UCDA and UCDB (compare fig.2) for inhomogeneous interfacial conductances and loading by a unit thermal gradient acting in $z$-direction $(d=200 \mu \mathrm{m}, \xi=0.34)$. 


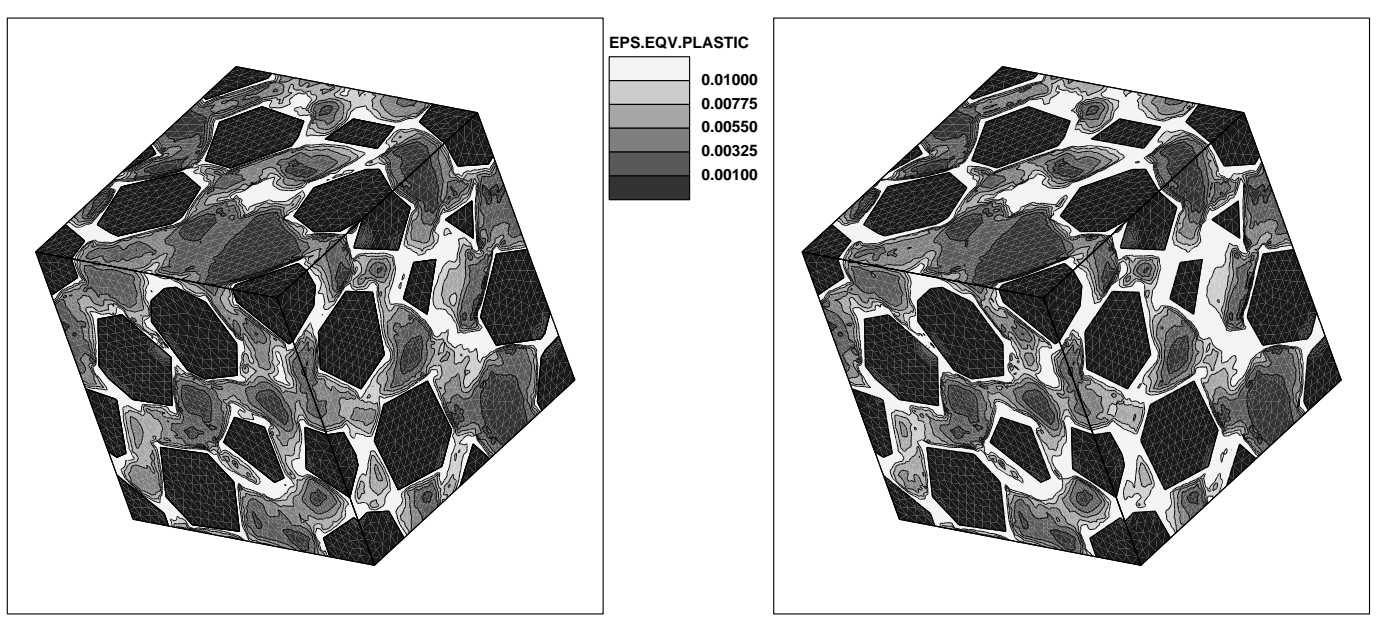

Fig. 6. Predicted distributions of equivalent plastic strain in the matrix of unit cell UCDA following cooling down from a stress-free temperature of $450 \mathrm{~K}$ to room temperature (left) and after subsequent heating up by $100 \mathrm{~K}$ (right).

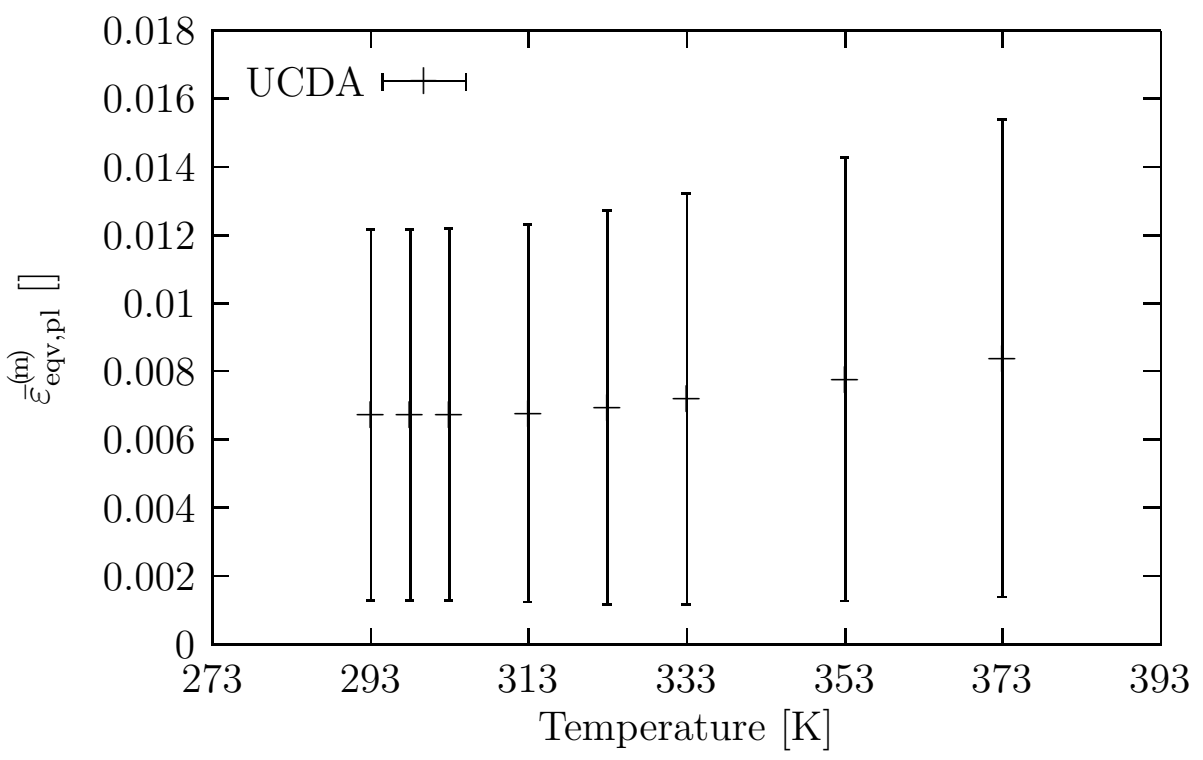

Fig. 7. Evolution of the average and the standard deviation of the accumulated equivalent plastic strain in the matrix, $\bar{\varepsilon}_{\text {eqv,pl }}^{(\mathrm{m})}$, during heating up from the cooled-down state to $373 \mathrm{~K}$ as predicted by unit cell $\mathrm{UCDA}(\xi=0.34)$. 


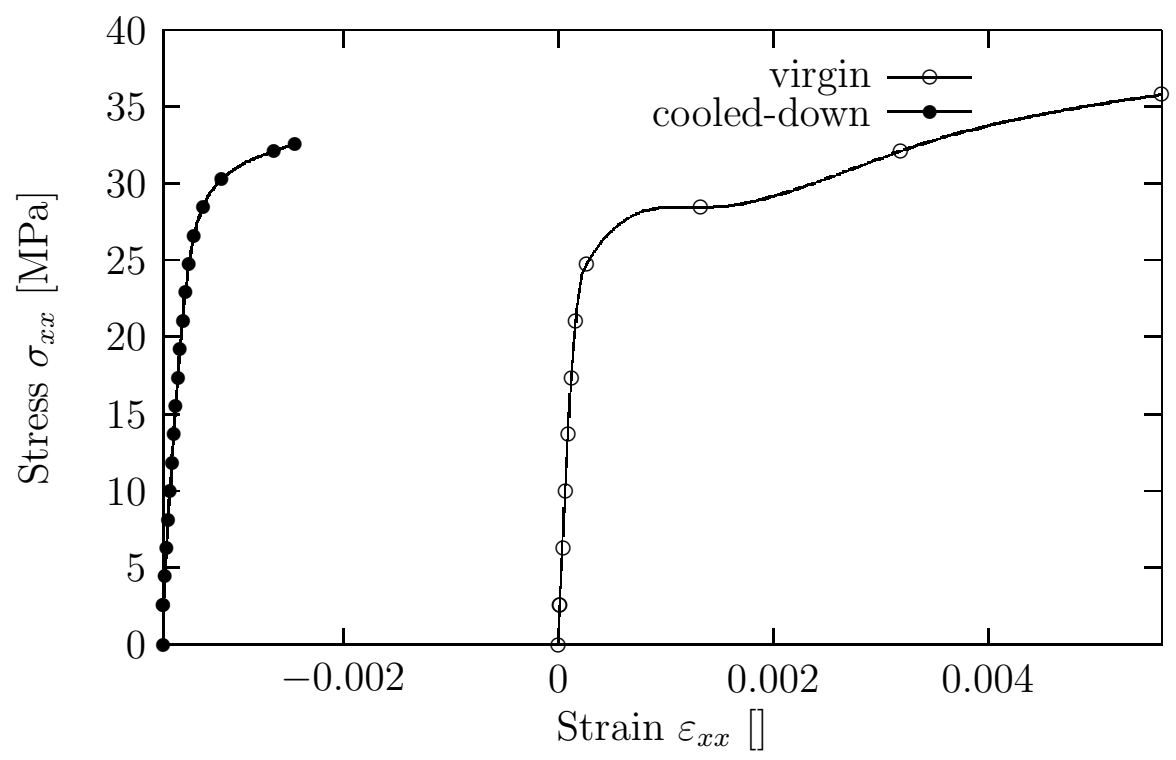

Fig. 8. Macroscopic stress-strain responses of DRMMC subjected to uniaxial tensile loading predicted for virgin and cooled-down conditions with unit cell UCDA ( $\xi=$ $0.34)$. 